\title{
Stent implantation into intracranial part of internal carotid artery in a patient with recurrent stroke, atrial fibrillation and iatrogenic bleeding during anticoagulant therapy
}

\author{
Anetta Lasek-Bal', Damian Ziaja², Krzysztof Ziaja² \\ 1Department of Neurology, Hospital No. 7 Medical University of Silesia, Professor Leszek Giec Medical Centre of Upper Silesian, \\ Katowice, Poland \\ 2Department of General and Vascular Surgery and Angiology, Hospital No. 7 Medical University of Silesia, Professor Leszek Giec Medical \\ Centre of Upper Silesian, Katowice, Poland
}

Postep Kardiol Inter 2013; 9, 1 (31): 75-78

DOI: 10.5114/pwki.2013.34030

\begin{abstract}
Cardiovascular diseases are the leading cause of stroke. We present a 45 -year-old woman with left hemisphere stroke, in which two sources of cerebral embolism were detected: atrial fibrillation and stenosis of intracranial part of left internal carotid artery. Oral anticoagulant therapy was used. The patient was qualified for intracranial revascularization. During anticoagulant therapy iatrogenic bleeding and then left hemisphere stroke occurred. Ultimately, the stent was implanted into the intracranial part of the internal carotid artery. Due to atrial fibrillation the patient is qualified for percutaneous closure of the left atrial appendage.
\end{abstract}

Key words: stent, intracranial carotid artery stenosis, stroke.

\section{Introduction}

Atherosclerosis of the carotid and vertebral arteries and heart diseases are responsible for over $50 \%$ of all ischemic strokes [1, 2]. Their role may be even more significant in view of the fact that in approximately $20 \%$ of strokes it is impossible to clearly define the cause of the incident $[3,4]$. Atrial fibrillation (AF) increases the risk of stroke around 5-fold especially in the elderly and in women [5-7]. Female sex is a negative factor in the $\mathrm{CHA}_{2} \mathrm{DS}_{2} \mathrm{VaSc}$ scale used to determine the annual risk of stroke in patients with atrial fibrillation. Stroke prevention is the main goal of treatment in patients with atrial fibrillation due to the fact that cerebrovascular incidents account for approximately $90 \%$ of complications of this arrhythmia. In patients with AF it is recommended to use oral anticoagulants $(\mathrm{OAC})$ after taking into account the risk of iatrogenic bleeding according to the HASBLED scale.

Arterial stenosis leading to arterio-arterial thrombi and hemodynamic insufficiency of the intracranial circulation occur less often in women than in men. Women are also less likely to benefit from endovascular treatment in the secondary prevention of stroke and therefore this treatment is less frequently considered in this group of patients after consideration of other potential risk factors of stroke. Epidemiologic data show that a cardiogenic mechanism is the main cause of ischemic stroke in women $[8,9]$.

\section{Aim}

We present a case of a young woman with stroke of the left hemisphere of the brain. She was also diagnosed with atrial fibrillation and $>70 \%$ stenosis of the cavernous part of the left internal carotid artery over a distance of $7 \mathrm{~mm}$. The secondary prevention of stroke included OAC. The patient was initially qualified for intracranial revascularization. Further clinical course of the patient has prompted us to modify the management regarding both the antithrombotic treatment and the arterial stenosis.

\section{Case report}

A 45-year-old female patient was admitted to the Department of Neurology due to sudden onset of speech distur-

Corresponding author:

Anetta Lasek-Bal MD, PhD, Department of Neurology, Hospital No. 7 Medical University of Silesia, Professor Leszek Giec Medical Centre of Upper Silesian, 45/47 Ziołowa St, 40-635 Katowice, Poland, tel.: +48 32359 83 06, +48 502695 389, fax: +48 32 202 95 92, e-mail: alasek@gcm.pl Received: 6.01.2013, accepted: 14.01.2013. 
bance and weakness of right limbs. The physical examination revealed mixed aphasia and right-hand hemiparesis ( 6 points on the National Institutes of Health and Stroke Scale NIHSS). The patient had a 3-month history of transient right upper extremities weakness with no other medical history. Computed tomography (CT) performed on the $1^{\text {st }}$ day did not reveal any changes. Intravenous thrombolysis was used to treat the acute stroke with rapid cessation of neurological symptoms. A control CT scan performed on the $2^{\text {nd }}$ day of stroke demonstrated the presence of an acute ischemic focus in the left hemisphere. The diagnosis of the causes of stroke revealed paroxysmal atrial fibrillation and left internal carotid artery stenosis ( $>70 \%$ over a distance of $7 \mathrm{~mm}$ in the cavernous part). Besides that, there were no significant changes in laboratory results including the assessment of antinuclear and anticardiolipin antibodies, antithrombin III, protein $\mathrm{C}$ and $\mathrm{S}$, coagulation parameters, glycemia, lipid profile as well as hepatic and renal parameters. There were also no abnormalities on transesophageal echocardiography and on angiography of the aortic arch, of the other carotid arteries and of the cerebral arteries. The secondary stroke prevention included rivaroxaban $20 \mathrm{mg} /$ day $\left(\mathrm{CHA}_{2} \mathrm{DS}_{2} \mathrm{VaSc}\right.$ 4 points, HASBLED 2 points). Stent implantation into the stenosed carotid artery was planned in 4 weeks time. Eventually the date of endovascular treatment was deferred at the patient's request for non-medical reasons. Three months after the stroke and after initiation of OAC the patient suffered from vaginal bleeding, which was accompanied by speech disturbances caused by another incident of stroke of the left hemisphere confirmed by CT scan of the brain. The patient was re-hospitalized in the Stroke Unit (4 points on the NIHSS scale), where due to anemia she received 2 units of packed red bloods. The OAC was discontinued and treatment with aspirin in modified doses was started (finally $100 \mathrm{mg} /$ day). The consulting gynecologist suggested an iatrogenic cause of bleeding. The neurologic status of the patient improved, but motor aphasia persisted, which significantly influenced the verbal contact with

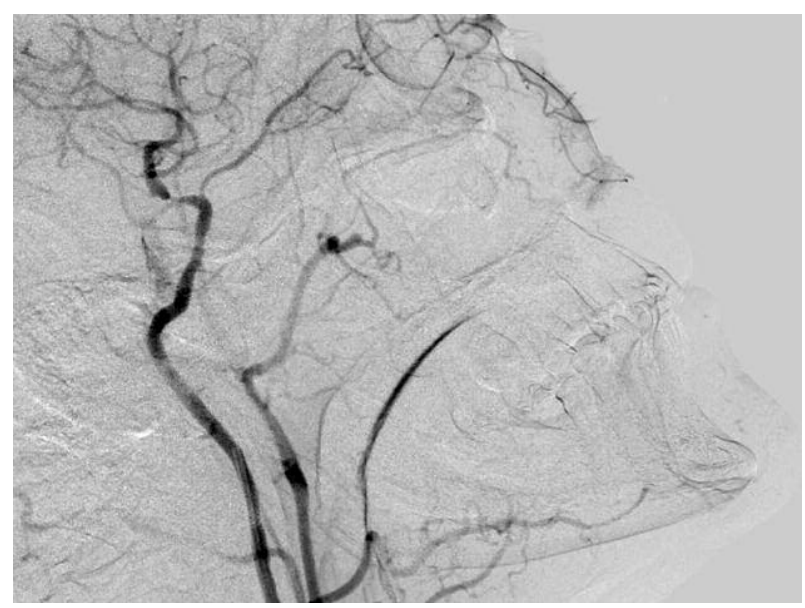

Fig. 1. Left internal carotid artery stenosis (cavernous part) the patient. In the $3^{\text {rd }}$ week after the second stroke, with stabilization of the general condition and normalization of morphology, the patient underwent stent implantation into the intracranial part of the left internal carotid artery. Arterial access was obtained through the right femoral artery. The area was locally anesthetized with $0.5 \%$ lignocaine solution, a $6 \mathrm{~F} 11 \mathrm{~cm}$ long sheath was introduced and $5000 \mathrm{IU}$ of unfractionated heparin was administered. The common carotid artery was engaged with a $0.35 \mathrm{~F}$ hydrophilic guidewire and VTK type $5 \mathrm{~F} 110 \mathrm{~cm}$ long catheter. To achieve stable access the $6 \mathrm{~F} 11 \mathrm{~cm}$ long sheath was subsequently changed for a $90 \mathrm{~cm}$ long one. The site of stenosis and its degree were assessed in detail on fluoroscopy after administration of contrast medium (Figure 1). The site of stenosis was accessed with a 0.014" (300 cm long) guidewire, which was further introduced distally to the stenosis using a VER $4 \mathrm{~F}$ catheter $(130 \mathrm{~cm}$ long). This was followed by the introduction and dilation of the Omega Monorail $3 \times 20 \mathrm{~mm}$ stent at the site of the lesion. Because of the persisting stenosis and inadequate stent expansion a $2 \times 20 \mathrm{~mm}$ balloon was used to improve the patency of the artery (Figure 2). Continuous infusion of heparin (20 $000 \mathrm{IU} / 24 \mathrm{~h}$ ) under aPTT control was used during the first day after the procedure. The patient was discharged on the $3^{\text {rd }}$ day after stent implantation in a neurological condition similar to the one present during admission to the hospital. She also received dual antiplatelet therapy (clopidogrel and aspirin $75 \mathrm{mg} /$ day). She remains under neurologic, cardiologic and surgical follow-up. Because of the presence of atrial fibrillation and history of antithrombotic treatment complications the patient is considered for percutaneous closure of the left atrial appendage (LAA) for prevention of cardiogenic stroke.

\section{Discussion}

The embolic mechanism of stroke was suspected in the presented case. It is difficult to determine whether the first

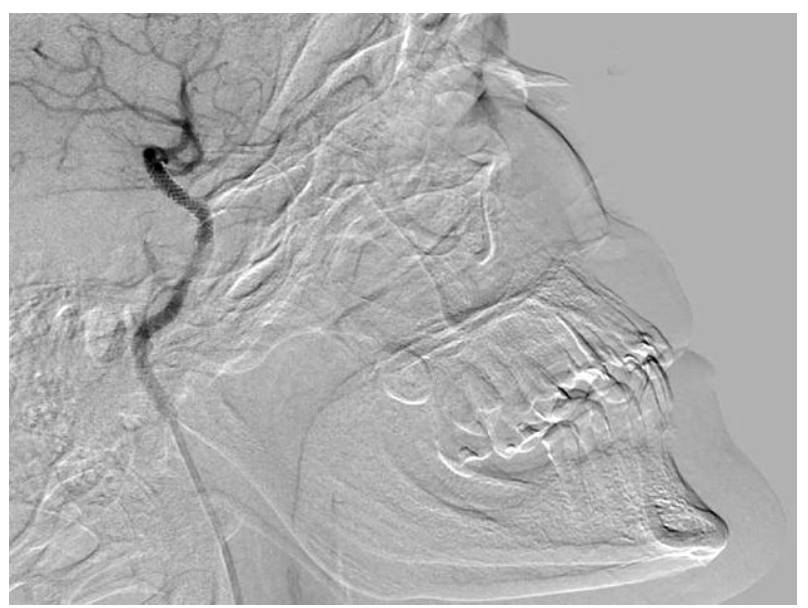

Fig. 2. Stent implanted in cavernous part of left internal carotid artery 
incident more likely had cardio-embolic or arterio-arterial origin. The left hemisphere of the brain is more often affected by ischemia, including in patients without significantly disturbed patency of the supplying arteries. It should be noted, however, that the presence of recurrent right upper extremity weakness in the period preceding stroke is equivalent to transient ischemic attacks of the left hemisphere of the brain. These homogeneous symptoms suggest the pathogenic role of carotid artery stenosis. However, the presence of atrial fibrillation might have been an independent source of cerebral embolism. Images of cerebral structures on CT did not reveal the presence of multifocal changes in the localization typical for cardiogenic origin, which however does not exclude this possibility. The course of the first stroke was favorable. Because of the annual risk of recurrent stroke (4\% according to the $\mathrm{CHA}_{2} \mathrm{DS}_{2} \mathrm{VaSc}$ score) and moderate risk of iatrogenic bleeding the therapy with $\mathrm{OAC}$ was initiated. Because of lack of overt risk factors of atherosclerosis, an atypical localization of arterial stenosis and the presence of an isolated lesion without other clinical and ultrasound signs of atherosclerosis, which has raised concerns regarding the atherogenic character of the stenosis, the patient has been cautiously qualified for endovascular treatment. The frequency of hemodynamically significant arterial stenosis in the intracranial area is estimated to be 8-9\% of all cases of stroke [10]. It mostly involves patients with generalized atherosclerosis. An inflammatory origin of carotid artery stenosis or fibro-muscular dysplasia was also taken into consideration in the present case. The second stroke occurred during vaginal bleeding, which according to the consulting gynecologist had an iatrogenic character. Anemia was undoubtedly an additional parameter (independent of hemodynamic parameters) worsening the perfusion of the left hemisphere of the brain. In view of the ineffectiveness of the existing secondary prevention of stroke and its side effects the patient was referred for revascularization of the intracranial part of the carotid artery, which was performed in the $3^{\text {rd }}$ week of the second ischemic stroke. The primary objective of this procedure was to improve the blood supply to the left hemisphere of the brain and to minimize the risk of subsequent ischemic strokes. The function of speech did not improve in the short term after the procedure. However, given the young age of the patient, the compensatory capabilities of the brain and improvement of hemodynamic parameters of the left hemisphere of the brain after the procedure, it is possible that speech disorders will attenuate in time.

Neurovascular procedures in patients with ischemic stroke have been a subject of individual reports and a few prospective studies [11-14]. Their results led to formulation of recommendations for such treatment in patients with symptomatic stenosis of intracranial arteries $>70 \%$ (class $\mathrm{Ilb}$, level of evidence $\mathrm{C}$ ). The most serious complications of intracranial revascularization in the first 30 days include stroke and death observed in $4.5-6.6 \%$ of patients $[11,13]$.
The clinical course of the presented patient during the first month after endovascular intervention was successful. The patient is on dual antiplatelet therapy. Speech therapy is being conducted. Due to the risk of cardiogenic cerebral embolism in the course of atrial fibrillation, closure of the left atrial appendage is considered in the later stage. The past experience with the use of OAC in the patient indicates insufficient efficacy and serious side effects of this form of therapy. Left atrial appendage occlusion would allow the most frequent locus of thrombus formation in the heart to be eliminated and antiplatelet monotherapy to be maintained in the secondary prevention of stroke.

In conclusion, it should be noted that the presented patient with ischemic stroke had two sources of cerebral embolism posing a serious risk of recurrent cerebral ischemia: atrial fibrillation and stenosis in the cavernous segment of the internal carotid artery. The pharmacological control of cardiogenic embolism proved insufficient and was complicated by iatrogenic bleeding. The decision to revascularize the intracranial part of the carotid artery to prevent subsequent strokes of the left hemisphere of the brain was taken by a team of physicians.

\section{References}

1. Grau AJ, Weimar C, Buggle F, et al. Risk factors, outcome, and treatment in subtypes of ischemic stroke: the German stroke data bank. Stroke 2001; 32: 2559-2566.

2. Valdueza J, Schreiber S, Roehl JE, Klingebiel R. Pathogenesis of stroke. Neurosonology and Neuroimaging of stroke. Thieme Publishing Group, Stuttgart- New York 2008.

3. Putaala J, Metso AJ, Metso TM, et al. Analysis of 1008 consecutive patients aged 15 to 49 with first-ever ischemic stroke: the Helsinki young stroke registry. Stroke 2009; 40: 1195-1203.

4. Cerrato $P$, Grasso M, Imperiale $D$, et al. Stroke in young patients: etiopathogenesis and risk factors in different age classes. Cerebrovasc Dis 2004; 18: 154-159.

5. Furberg CD, Psaty BM, Manolio T, et al. Prevalence of atrial fibrillation in elderly subjects (the Cardiovascular Health Study). Am J Cardiol 1994; 74: 236-241.

6. Go AS, Hylek EM, Phillips KA, et al. Prevalence of diagnosed atrial fibrillation in adults: national implications for rhythm management and stroke prevention: the AnTicoagulation and Risk Factors in Atrial Fibrillation (ATRIA) Study. JAMA 2001; 285: 2370-2375.

7. Humphries KH, Kerr CR, Connolly SJ, et al. New-onset atrial fibrillation: sex differences in presentation, treatment, and outcome. Circulation 2001; 103: 2365-2370.

8. Förster A, Gass A, Kern R, et al. Gender differences in acute ischemic stroke: etiology, stroke patterns and response to thrombolysis. Stroke 2009; 40: 2428-2432.

9. Reeves MJ, Bushnell CD, Howard G, et al. Sex differences in stroke: epidemiology, clinical presentation, medical care, and outcomes. Lancet Neurol 2008; 7: 915-926.

10. Meyers PM, Schumacher HC, Higashida RT, et al. Indications for the performance of intracranial endovascular neurointerventional procedures: a scientific statement from the American Heart Association Council on Cardiovascular Radiology and Intervention, Stroke Council, Council on Cardiovascular Surgery and Anesthesia, Interdisciplinary Council on Peripheral Vascular Disease, and Interdis- 
ciplinary Council on Quality of Care and Outcomes Research. Circulation 2009; 119: 2235-2249.

11. SSYLVIA Study Investigators. Stenting of Symptomatic Atherosclerotic Lesions in the Vertebral or Intracranial Arteries (SSYLVIA): study results. Stroke 2004; 35: 1388-1392.

12. Henkes H, Miloslavski E, Lowens S, et al. Treatment of intracranial atherosclerotic stenoses with balloon dilatation and self-expanding stent deployment (WingSpan). Neuroradiology 2005; 47: 222-228.
13. Fiorella D, Levy EI, Turk AS, et al. US multicenter experience with the Wingspan stent system for the treatment of intracranial atheromatous disease: periprocedural results. Stroke 2007; 38: 881-887.

14. Gupta R, Al-Ali F, Thomas AJ, et al. Safety, feasibility, and short-term follow-up of drug-eluting stent placement in the intracranial and extracranial circulation. Stroke 2006; 37: 2562-2566. 